Bull. Austral. Math. Soc.

05E15, 11L05, 11T23, 20G40

VOL. 63 (2001) [15-20]

\title{
A COUNTING FORMULA ABOUT THE SYMPLECTIC SIMILITUDE GROUP
}

\section{KWANKYU LEe}

We derive an explicit formula for the number of elements in the symplectic similitude group GSp $(2 n, q)$ with given trace and determinant.

\section{INTRODUCTION}

Let $\mathbb{F}_{q}$ be the finite field with $q$ elements: Recall that the symplectic similitude group $\operatorname{GSp}(2 n, q)$ over $\mathbb{F}_{q}$ is defined by

$$
\mathrm{GSp}(2 n, q)=\left\{\left.g \in \mathrm{GL}(2 n, q)\right|^{t} g J g=\alpha(g) J \text { for some } \alpha(g) \in \mathbb{F}_{q}^{\times}\right\},
$$

where $J$ denotes $\left[\begin{array}{cc}0 & 1_{n} \\ -1_{n} & 0\end{array}\right]$. This paper addresses the problem of counting the number of elements in $\operatorname{GSp}(2 n, q)$ with given trace and determinant. More formally, we want to find the value of

$$
C(\zeta, \eta)=|\{g \in \mathrm{GSp}(2 n, q) \mid \operatorname{det} g=\zeta, \operatorname{tr} g=\eta\}|,
$$

when $\zeta \in \mathbb{F}_{q}^{\mathbf{x}}, \eta \in \mathbb{F}_{q}$ are given. In [1], Kim gave a related result: an explicit formula for the number of elements in $\operatorname{GSp}(2 n, q)$ with given trace. In this paper, we derive an explicit formula for $C(\zeta, \eta)$.

TheOREM 1. Let $\zeta \in \mathbb{F}_{q}^{\times}, \eta \in \mathbb{F}_{q}$. Let $S$ denote the number of $n$-th roots of $\zeta$ in $\mathbb{F}_{q}$, and let

$$
T_{m}=q \sum_{\alpha \in \mathbb{F}_{q}^{\times}} \sum_{\alpha_{1}, \ldots, \alpha_{m} \in \mathbf{F}_{q}^{\times}} t\left(\alpha^{n}, \alpha_{1}+\alpha \alpha_{1}^{-1}+\cdots+\alpha_{m}+\alpha \alpha_{m}^{-1}\right)-(q-1)^{m} S,
$$

where $t(x, y)=1$ if $(x, y)=(\zeta, \eta), 0$ otherwise; and the inner sum is regarded as $t\left(\alpha^{n}, 0\right)$ for $m=0$. Then we have

$$
\begin{array}{r}
C(\zeta, \eta)=q^{n^{2}-1} \sum_{b=0}^{[n / 2]}\left(q^{b^{2}+b}\left[\begin{array}{c}
n \\
2 b
\end{array}\right] \prod_{q}^{b}\left(q^{2 j-1}-1\right) \sum_{l=0}^{[(n / 2)-b]} q^{l} R(n-2 b+1, l) T_{n-2 b-2 l}\right) \\
+q^{n^{2}-1} \prod_{j=1}^{n}\left(q^{2 j}-1\right) S,
\end{array}
$$

Received 11th January, 2000

This work was supported by the Brain Korea 21 Project.

Copyright Clearance Centre, Inc. Serial-fee code: 0004-9727/01 SA2.00+0.00. 
where $R(m, l)$ denotes $\sum_{0<j_{1}<\cdots<j_{l}<m-l} \prod_{\nu=1}^{l}\left(q^{m-\nu-j_{\nu}}-1\right)$ with $R(m, 0)=1$.

The definition of the $q$-binomial coefficient $\left[\begin{array}{l}n \\ b\end{array}\right]_{q}$ is given in the next section.

\section{Preparation}

Recall that the symplectic group over $\mathbb{F}_{q}$ is defined by

$$
\mathrm{Sp}(2 n, q)=\left\{\left.g \in \mathrm{GL}(2 n, q)\right|^{t} g J g=J\right\} .
$$

Observe that

$$
\operatorname{GSp}(2 n, q)=\coprod_{\alpha \in \mathbb{P}_{q}^{x}} d_{\alpha} \operatorname{Sp}(2 n, q)
$$

with $d_{\alpha}=\left[\begin{array}{cc}1_{n} & 0 \\ 0 & \alpha 1_{n}\end{array}\right]$. A maximal parabolic subgroup $P$ of $\mathrm{Sp}(2 n, q)$ is given by

$$
P=P(2 n, q)=\left\{\left[\begin{array}{cc}
A & 0 \\
0 & { }^{t} A^{-1}
\end{array}\right]\left[\begin{array}{cc}
1_{n} & B \\
0 & 1_{n}
\end{array}\right] \mid A \in \mathrm{GL}(n, q),{ }^{t} B=B\right\} .
$$

We let, for $0 \leqslant b \leqslant n$,

$$
A_{b}=A_{b}(2 n, q)=\left\{g \in P(2 n, q) \mid \sigma_{b} g \sigma_{b}^{-1} \in P(2 n, q)\right\},
$$

where

$$
\sigma_{b}=\left[\begin{array}{cccc}
0 & 0 & 1_{b} & 0 \\
0 & 1_{n-b} & 0 & 0 \\
-1_{b} & 0 & 0 & 0 \\
0 & 0 & 0 & 1_{n-b}
\end{array}\right]
$$

Now the Bruhat decomposition of $\operatorname{Sp}(2 n, q)$ with respect to $P$ says

$$
\mathrm{Sp}(2 n, q)=\coprod_{b=0}^{n} P \sigma_{b} P=\coprod_{b=0}^{n} P \sigma_{b}\left(A_{b} \backslash P\right) .
$$

This decomposition will play a crucial role in our proof of the theorem.

Let $g_{n}$ be the number of $n \times n$ nonsingular matrices over $\mathbb{F}_{q}$, and $a_{n}$ the number of $n \times n$ nonsingular alternating matrices over $\mathbb{F}_{q}$. We define $g_{0}=a_{0}=1$ for convenience. 
Then

$$
\begin{aligned}
g_{n} & =\prod_{j=0}^{n-1}\left(q^{n}-q^{j}\right)=q^{\left(n^{2}-n\right) / 2} \prod_{j=1}^{n}\left(q^{j}-1\right), \\
a_{n} & = \begin{cases}q^{(n / 2)((n / 2)-1)} \prod_{j=1}^{n / 2}\left(q^{2 j-1}-1\right) & \text { for } n \text { even, } \\
0 & \text { for } n \text { odd, }\end{cases} \\
\left|A_{b} \backslash P\right| & =q^{\left(b^{2}+b\right) / 2}\left[\begin{array}{l}
n \\
b
\end{array}\right]_{q} .
\end{aligned}
$$

The $q$-binomial coefficient $\left[\begin{array}{l}n \\ r\end{array}\right]_{q}$ is defined by

$$
\left[\begin{array}{l}
n \\
r
\end{array}\right]_{q}=\prod_{j=0}^{r-1} \frac{q^{n-j}-1}{q^{r-j}-1}
$$

See [1] and [2] for more details of these facts.

\section{PROOF OF THE THEOREM}

For any complex-valued function $f$ defined on $\mathbb{F}_{q}$ and $\sigma, \tau \in \mathbb{F}_{q}$, let $M_{m}(f ; \sigma ; \tau)$ denote

$$
\sum_{\alpha_{1}, \ldots, \alpha_{m} \in \mathbf{F}_{q}^{\times}} f\left(\sigma \alpha_{1}+\tau \alpha_{1}^{-1}+\cdots+\sigma \alpha_{m}+\tau \alpha_{m}^{-1}\right)
$$

with $M_{0}(f ; \sigma, \tau)=f(0)$. Remember that $R(m, l)$ was defined in Theorem 1 .

LEMMA 1. Let $f$ be an arbitrary complex-valued function defined on $\mathbb{F}_{q}$, and $\sigma, \tau \in$ $\mathbb{F}_{q}^{\times}$. Then

$$
\begin{aligned}
& \sum_{g \in \mathrm{GL}(n, q)} f\left(\sigma \operatorname{tr} g+\tau \operatorname{tr} g^{-1}\right) \\
& =q^{\left(n^{2}-n\right) / 2-1} \sum_{l=0}^{[n / 2]} q^{l} R(n+1, l)\left(q M_{n-2 l}(f ; \sigma, \tau)-(q-1)^{n-2 l} \sum_{\gamma \in \mathbb{F}_{q}} f(\gamma)\right) \\
& +q^{\left(n^{2}-n\right) / 2-1} \prod_{j=1}^{n}\left(q^{j}-1\right) \sum_{\gamma \in \mathbb{F}_{q}} f(\gamma) .
\end{aligned}
$$

Proof: Recall that for a nontrivial additive character $\lambda$ of $\mathbf{F}_{q}$ and $\sigma, \tau \in \mathbb{F}_{q}$, the ordinary Kloosterman sum $K(\lambda ; \sigma, \tau)$ is defined by $K(\lambda ; \sigma, \tau)=\sum_{\alpha \in \mathbb{F}_{q}^{x}} \lambda\left(\sigma \alpha+\tau \alpha^{-1}\right)$. First we state a slightly modified version of Theorem 4.3 in [2]. 
SuBlemMA. Let us define $K_{\mathrm{GL}(n, q)}(\lambda ; \sigma, \tau)=\sum_{g \in \mathrm{GL}(n, q)} \lambda\left(\sigma \operatorname{tr} g+\tau \operatorname{tr} g^{-1}\right)$ for a nontrivial additive character $\lambda$ of $\mathrm{F}_{q}$ and $\sigma, \tau \in \mathbf{F}_{q}^{\times}$. Then

$$
K_{\mathrm{GL}(n, q)}(\lambda ; \sigma, \tau)=q^{\left(n^{2}-n\right) / 2} \sum_{l=0}^{[n / 2]} q^{l} R(n+1, l) K(\lambda ; \sigma, \tau)^{n-2 l} .
$$

Now pick a nontrivial additive character $\lambda$ of $\mathbb{F}_{q}$. We then have

$$
\begin{aligned}
\sum_{g \in \mathrm{GL}(n, q)} & f\left(\sigma \operatorname{tr} g+\tau \operatorname{tr} g^{-1}\right) \\
& =\sum_{\gamma \in \mathbb{F}_{q}}\left|\left\{g \in \mathrm{GL}(n, q) \mid \sigma \operatorname{tr} g+\tau \operatorname{tr} g^{-1}=\gamma\right\}\right| f(\gamma) \\
& =\frac{1}{q} \sum_{\gamma \in \mathbb{F}_{q}} \sum_{\delta \in \mathbb{F}_{q}} \sum_{g \in \mathrm{GL}(n, q)} \lambda\left(\delta\left(\sigma \operatorname{tr} g+\tau \operatorname{tr} g^{-1}-\gamma\right)\right) f(\gamma) \\
& =\frac{1}{q} \sum_{\gamma \in \mathbb{F}_{q}} \sum_{\delta \in \mathbb{F}_{q}^{\times}} K_{\mathrm{GL},(n, q)}(\lambda ; \delta \sigma, \delta \tau) \lambda(-\delta \gamma) f(\gamma)+\frac{1}{q} g_{n} \sum_{\gamma \in \mathbb{F}_{q}} f(\gamma) .
\end{aligned}
$$

By the sublemma,

$$
\begin{aligned}
& \sum_{g \in \mathrm{GL}(n, q)} f\left(\sigma \operatorname{tr} g+\tau \operatorname{tr} g^{-1}\right) \\
& =q^{\left(n^{2}-n\right) / 2} \sum_{l=0}^{[n / 2]} q^{l} R(n+1, l) \frac{1}{q} \sum_{\gamma \in \mathbb{F}_{q}} \sum_{\delta \in \mathbf{F}_{q}^{x}} K(\lambda ; \delta \sigma, \delta \tau)^{n-2 l} \lambda(-\delta \gamma) f(\gamma) \\
& +q^{\left(n^{2}-n\right) / 2-1} \prod_{j=1}^{n}\left(q^{j}-1\right) \sum_{\gamma \in \mathbf{F}_{q}} f(\gamma) .
\end{aligned}
$$

But we have

$$
\begin{aligned}
& \frac{1}{q} \sum_{\gamma \in \mathbb{F}_{q}} \sum_{\delta \in \mathbf{F}_{q}^{\times}} K(\lambda ; \delta \sigma, \delta \tau)^{n-2 t} \lambda(-\delta \gamma) f(\gamma) \\
& =\frac{1}{q} \sum_{\gamma \in \mathbb{F}_{q}} \sum_{\delta \in \mathbb{F}_{q}^{\times}}\left(\sum_{\alpha \in \mathbb{F}_{q}^{\times}} \lambda\left(\delta \sigma \alpha+\delta \tau \alpha^{-1}\right)\right)^{n-2 l} \lambda(-\delta \gamma) f(\gamma) \\
& =\frac{1}{q} \sum_{\gamma \in \mathbb{F}_{q}} \sum_{\delta \in \mathbb{F}_{q}} \sum \lambda\left(\delta\left(\sigma \alpha_{1}+\tau \alpha_{1}^{-1}+\cdots+\sigma \alpha_{n-2 l}+\tau \alpha_{n-2 l}^{-1}-\gamma\right)\right) f(\gamma) \\
& -\frac{1}{q}(q-1)^{n-2 l} \sum_{\gamma \in \mathbb{F}_{q}} f(\gamma) \\
& =\sum f\left(\sigma \alpha_{1}+\tau \alpha_{1}^{-1}+\cdots+\sigma \alpha_{n-2 l}+\tau \alpha_{n-2 l}^{-1}\right)-\frac{1}{q}(q-1)^{n-2 l} \sum_{\gamma \in \mathbf{F}_{q}} f(\gamma),
\end{aligned}
$$

where the unspecified sums are taken over $\alpha_{1}, \ldots, \alpha_{n-2 l} \in \mathbb{F}_{q}^{\times}$. Thus we get the lemma. 
Lemia 2. Let $e, f$ be arbitrary complex-valued functions defined on $\mathbf{F}_{q}$. Then

$$
\begin{aligned}
\sum_{g \in \mathrm{GSp}(2 n, q)} e(\operatorname{det} g) & f(\operatorname{tr} g) \\
= & q^{n^{2}-1} \sum_{b=0}^{[n / 2]}\left(q^{b^{2}+b}\left[\begin{array}{c}
n \\
2 b
\end{array}\right] \prod_{q}^{b}\left(q^{2 j-1}-1\right) \sum_{l=0}^{[(n / 2)-b]} q^{l} R(n-2 b+1, l)\right. \\
& \left.\times \sum_{\alpha \in \mathbb{F}_{q}^{X}} e\left(\alpha^{n}\right)\left(q M_{n-2 b-2 l}(f ; 1, \alpha)-(q-1)^{n-2 b-2 l} \sum_{\gamma \in \mathbb{F}_{q}} f(\gamma)\right)\right) \\
& +q^{n^{2}-1} \prod_{j=1}^{n}\left(q^{2 j}-1\right) \sum_{\alpha \in \mathbb{F}_{q}^{\times}} e\left(\alpha^{n}\right) \sum_{\gamma \in \mathbb{F}_{q}} f(\gamma)
\end{aligned}
$$

Proof: We have

$$
\begin{aligned}
\sum_{g \in \mathrm{GSp}(2 n, q)} e(\operatorname{det} g) f(\operatorname{tr} g) & =\sum_{\alpha \in \mathbb{P}_{q}^{\times}} \sum_{g \in \operatorname{Sp}(2 n, q)} e\left(\operatorname{det}\left(d_{\alpha} g\right)\right) f\left(\operatorname{tr}\left(d_{\alpha} g\right)\right) \\
& =\sum_{\alpha \in \mathbb{F}_{q}^{\times}} e\left(\alpha^{n}\right) \sum_{g \in \operatorname{Sp}(2 n, q)} f\left(\operatorname{tr}\left(d_{\alpha} g\right)\right) .
\end{aligned}
$$

By the Bruhat decomposition,

$$
\sum_{g \in \operatorname{GSp}(2 n, q)} e(\operatorname{det} g) f(\operatorname{tr} g)=\sum_{\alpha \in \mathbb{F}_{q}^{\times}} e\left(\alpha^{n}\right) \sum_{b=0}^{n}\left|A_{b} \backslash P\right| \sum_{g \in P} f\left(\operatorname{tr}\left(d_{\alpha} g \sigma_{b}\right)\right) .
$$

Observe that the structure of $P$ allows us to compute explicitly $\operatorname{tr}\left(d_{\alpha} g \sigma_{b}\right)$ for $g \in P$. Thus we get

$$
\begin{aligned}
\sum_{g \in \mathrm{GSp}(2 n, q)} e(\operatorname{det} g) f(\operatorname{tr} g) & \\
=\sum_{\alpha \in \mathbb{P}_{q}^{\mathrm{X}}} e\left(\alpha^{n}\right) \sum_{b=0}^{n} & \left|A_{b} \backslash P\right|\left(q^{\left(n^{2}+n\right) / 2-1}\left(g_{n}-a_{b} g_{n-b} q^{b(n-b)}\right) \sum_{\gamma \in \mathbb{F}_{q}} f(\gamma)\right. \\
& \left.+a_{b} q^{\left(n^{2}+n\right) / 2+b(n-b)} \sum_{g \in \mathrm{GL}(n-b, q)} f\left(\operatorname{tr} g+\alpha \operatorname{tr} g^{-1}\right)\right) .
\end{aligned}
$$

Now use Lemma 1 to resolve the last expression. This completes the proof.

PROOF OF THEOREM 1: We obtain Theorem 1 from Lemma 2 simply by setting $e$ and $f$ to be the functions defined by

$$
e(\alpha)=\left\{\begin{array}{ll}
1 & \text { if } \alpha=\zeta, \\
0 & \text { otherwise }
\end{array} \text { and } f(\alpha)= \begin{cases}1 & \text { if } \alpha=\eta \\
0 & \text { otherwise }\end{cases}\right.
$$

for $\alpha \in \mathbf{F}_{q}$. 
REMARK. Tables of $C(\zeta, \eta)$ for $\operatorname{GSp}(2 n, q)$ with different $n$ and $q$ are included below. These were produced by a Mathematica program into which the formula for $C(\zeta, \eta)$ was coded. The referee explained the apparent symmetries in the tables by observing that $C(\zeta, \eta)=C\left(\alpha^{2 n} \zeta, \alpha \eta\right)$ for $\alpha \in \mathbf{F}_{q}^{x}$.

\section{TABLES OF $C(\zeta, \eta)$}

$\operatorname{GSp}(6,3)$

\begin{tabular}{c|ccc}
$C(\zeta, \eta)$ & $\eta=0$ & $\eta=1$ & $\eta=2$ \\
\hline$\zeta=1$ & 3053423790 & 3058639785 & 3058639785 \\
$\zeta=2$ & 3063934512 & 3053384424 & 3053384424
\end{tabular}

$\operatorname{GSp}(4,5)$

\begin{tabular}{c|ccccc}
$C(\zeta, \eta)$ & $\eta=0$ & $\eta=1$ & $\eta=2$ & $\eta=3$ & $\eta=4$ \\
\hline$\zeta=1$ & 3867500 & 3713125 & 3713125 & 3713125 & 3713125 \\
$\zeta=2$ & 0 & 0 & 0 & 0 & 0 \\
$\zeta=3$ & 0 & 0 & 0 & 0 & 0 \\
$\zeta=4$ & 3870000 & 3712500 & 3712500 & 3712500 & 3712500
\end{tabular}

$\operatorname{GSp}(6,5)$

\begin{tabular}{c|ccccc}
$C(\zeta, \eta)$ & $\eta=0$ & $\eta=1$ & $\eta=2$ & $\eta=3$ & $\eta=4$ \\
\hline$\zeta=1$ & 91408007812500 & 91395326171875 & 91401669921875 & 91401669921875 & 91395326171875 \\
$\zeta=2$ & 91395312500000 & 91408015625000 & 91395328125000 & 91395328125000 & 91408015625000 \\
$\zeta=3$ & 91395312500000 & 91395328125000 & 91408015625000 & 91408015625000 & 91395328125000 \\
$\zeta=4$ & 91408007812500 & 91401669921875 & 91395326171875 & 91395326171875 & 91401669921875
\end{tabular}

\section{REFERENCES}

[1] D.S. Kim, 'Exponential sums for symplectic groups and their applications', Acta Arith. 88 (1999), 155-171.

[2] D.S. Kim, 'Gauss sums for symplectic groups over a finite field', Monatsh. Math. 126 (1998), 55-71.

Department of Mathematics

Sogang University

Seoul 121-742

Korea

e-mail: leekk@math.sogang.ac.kr 